\title{
Efeitos da fisiomotricidade no risco e medo de quedas em idosas
}

\author{
Fisiomotricity effects on risk and fear of falling in elderly
}

\author{
Luan Antônio dos Santos da Silva ${ }^{1}$, Maria Claudia Gonçalves ${ }^{2}$, Sarah Tarcisia Rebelo \\ Ferreira de Carvalho ${ }^{2}$, Karla Virginia Bezerra de Castro Soares ${ }^{2}$
}

\begin{abstract}
Resumo: Introdução: Evidências epidemiológicas apontam que $32 \%$ da população idosa com idade entre 65 e 74 anos sofre queda pelo menos uma vez a cada ano. Este percentual aumenta para $35 \%$ nos idosos entre 75 e 84 anos e atinge os $51 \%$ naqueles acima de 85 anos. Objetivo: analisar os efeitos da Fisiomotricidade na minimização do risco de quedas e no medo de cair. Material e métodos: Estudo realizado com 30 idosas hígidas, do gênero feminino, com idade entre 70 e 75 anos, com capacidade física e cognitiva para realizar os testes previamente escolhidos e disponibilidade para participar da pesquisa. Foram aplicadas as escalas de Tinetti e Falls Efficacy Scale International (FES-1-BRASIL), para avaliar o risco e medo de cair respectivamente, ambos aplicados antes e após um programa de fisiomotricidade, realizado duas vezes por semana, por 50 minutos/dia, durante três meses. A análise estatística foi realizada por meio do programa SPSS 18.0, sendo considerado significativo $p<0,05$. Resultados: Para o risco de quedas antes e após a intervenção o índice de Tinetti apontou valores de 21 e 25 , com $z$ de $-4,80$ e $p=0,000$. Com relação ao medo de cair os valores de escores foram de 30 antes da intervenção e 21 após, com z de $-4,57$ e $p=0,000$. Conclusão: $O$ treinamento da Fisiomotricidade por meio do protocolo proposto neste estudo promoveu redução do risco e medo de cair das idosas estudadas, sugere-se que sejam feitos novos estudos clínicos com presença de grupo controle e maior número de participantes.
\end{abstract}

Palavras-chave: Risco de queda. Medo de cair. Idoso.

Abstract: Introduction: Epidemiological evidence show that 32\% of the elderly population aged between 65 and 74 years suffer fall at least once each year. This percentage increases to $35 \%$ in the elderly between 75 and 84 years and reaches $51 \%$ in those over 85 years. Objective: To analyze the effects of fisiomotricity to minimize the risk of falls and fear of falling. Methods: Study of 30 older otherwise healthy, female, aged 70 and 75 , with physical and cognitive ability to perform the tests previously chosen and availability to participate in the pesquisa. Used are the Tinetti and Falls Efficacy Scale International (FES 1-BRAZIL), to assess the risk and a fear of falling, respectively, applied both before and after a fisiomotricity program, performed twice a week for 50 minutes / day for three months. Statistical analysis was performed using SPSS 18.0, considering significant $p<0.05$. Results: For the risk of falls before and after the intervention Tinetti's index showed values of 21 and 25 with $z-4.80$ and $p=0.000$. Regarding the fear of falling scores values were 30 before the intervention and after 21, with $z-4.57$ and $p$ $=0.000$. Conclusion: The training fisiomotricity through the proposed protocol in this study promoted reducing the risk and fear of falling in elderly studied, it is suggested to be made new clinical studies with the presence of a control group and a larger number of participants.

Keywords: Risk of falling, Fear of falling, Aged .

1 - Graduado do Curso de Fisioterapia - UNICEUMA

2- Docentes - UNICEUMA

Rev. Investig, Bioméd. São Luís 7:2-11. 2015 


\section{Introdução}

O envelhecimento populacional é uma realidade no Brasil e no mundo e põe em destaque um importante problema de ordem clínica e social: a queda. Seja por sua frequência e morbidade, ou pelo elevado custo social e econômico de lesões delas decorrentes, as quedas constituem um relevante problema de saúde pública que pode ser evitado 1 .

Evidências epidemiológicas apontam que $32 \%$ da população idosa com idade entre 65 e 74 anos sofre queda pelo menos uma vez a cada ano. Este percentual aumenta para $35 \%$ nos idosos entre 75 e 84 anos e atinge os $51 \%$ naqueles acima de 85 anos. No Brasil, a estimativa é de que $20 \%$ a $30 \%$ desta população caia neste intervalo de tempo e 15 a $50 \%$ apresentem risco de morte no ano seguinte ou hospitalização. Entre os anos de 1996 e 2005, cerca de 24.645 idosos morreram por consequência de quedas, constituindo o terceiro lugar de mortalidade e o primeiro lugar entre as internações hospitalares ${ }^{2}$.

A queda, definida como um evento não intencional resultante da mudança de posição do indivíduo para um nível postural mais baixo em relação à sua posição inicial, geralmente ocorre por perda total do equilíbrio relacionado à insuficiência dos mecanismos neurais $e$ osteoarticulares envolvidos na manutenção da postura ${ }^{3}$.

Além dos problemas de ordem física, a queda também desencadeia o medo de cair que, se não percebido precocemente, pode progredir para a "Ptofobia", termo que surgiu a partir da identificação da síndrome pós-queda, e designa a reação fóbica em manter-se de pé ou andar, ou ainda, interagir socialmente ${ }^{4}$.

A atividade física pode ser considerada ferramenta retardatária no processo do envelhecimento neurofuncional estendendo-se a prevenção de quedas mediante diferentes mecanismos a exemplo do fortalecimento da musculatura efetora, melhora dos reflexos e da sinergia motora responsável pelas reações posturais, incremento da flexibilidade e mobilidade que repercutem em maior confiança e velocidade de marcha ${ }^{5}$.

Dentre as inúmeras opções de atividades físicas voltadas ao idoso, destaca-se a fisiomotricidade, protocolo cinesioterapêutico proposto por Castro ${ }^{6}$ e que tem por característica exercícios que garantem condições de mínimo estresse articular e níveis lentos de execução, em função de máximo controle motor durante os movimentos, aliados à constância da mentalização sobre os movimentos em execução.

Compreendendo a relevância do tema e entendendo que existem ainda lacunas acerca do que leva a um envelhecimento bem sucedido, o objetivo deste estudo foi analisar os efeitos de um protocolo de tratamento baseado na Fisiomotricidade na minimização do risco de quedas e no medo de cair.

\section{Material e métodos}

\section{Aspectos Éticos}

Este estudo foi desenvolvido respeitando as normas estabelecidas na Resolução № 466/2012 do Conselho Nacional de Saúde com relação à Realização de 
Pesquisa em Seres Humanos e aprovado pelo Comitê de Ética e Pesquisa (CEP), da Universidade CEUMA pelo parecer de número 614.887. Todos os participantes assinaram 0 Termo de Consentimento Livre e Esclarecido (TCLE) contendo informações sobre os processos, riscos e benefícios associados ao estudo.

\section{Amostra da Pesquisa}

A pesquisa foi realizado na cidade de São Luís, entre fevereiro e maio de 2014. A amostra foi selecionada através do sorteio das 80 fichas de inscrição de idosos, de ambos os sexos, interessados em participar do projeto Uniceuma sem fronteiras da Universidade Ceuma no ano de 2014, na cidade de São Luís. O projeto, que funciona desde agosto de 2010, oferece aos idosos da comunidade atividades que propiciam práticas de diferentes atividades físicas e fisioterapia, a exemplo da Fisiomotricidade, além de oficina de memória e inclusão digital para idosos.

Excluíram-se do estudo idosos que apresentavam doenças neurológicas, como acidente vascular encefálico, doença de Parkinson, distúrbios do aparelho vestibular, deficiência visual não corrigida, paralisias de qualquer etiologia e alterações ortopédicas, como amputações, fraturas, incapacidade de se manter em ortostatismo e de se locomover sem assistência, também os que apresentavam dores agudas ocasionais ou qualquer patologia que os impossibilitavam de realizar na íntegra os exercícios do protocolo ou que não obtiveram o mínimo de $75 \%$ de frequência nas aulas.

Desta forma, a amostra foi composta por 30 idosos hígidas, do gênero feminino, com idade entre 70 e 75, com capacidade física e cognitiva para realizar os testes previamente escolhidos e disponibilidade para participar da pesquisa.

\section{Instrumentos de avaliação}

Foram utilizadas as escalas validadas de Tinetti e o Falls Efficacy Scale International (FES-IBRASIL), em função de sua praticidade, fácil compreensão e execução.

A escala de Tinetti foi utilizada para avaliar o equilíbrio e a marcha de idosos correlacionandoos ao risco de cair e consiste na avaliação da execução de 16 tarefas, dentre elas citam-se transferência de sentado para de pé e giro de $360^{\circ}$. São atribuídos pontos de 0 a 2 na realização de cada tarefa, totalizando, no máximo, 28 pontos. O escore acima de 24 pontos significa um baixo risco de queda, entre 19 e 24 pontos significa um risco moderado e abaixo de 19 significa um alto risco de queda ${ }^{7,8}$. A Escala de Equilíbrio e Marcha de Tinetti foi aplicada conforme o modelo de Tinetti $^{9}$ o qual foi traduzido para o português e validado no Brasil por Gomes ${ }^{10}$.

Para a avaliação do medo de cair utilizou-se como instrumento o FES-I-BRASIL que é uma escala que apresenta questões sobre a preocupação com a possibilidade de cair ao realizar 16 atividades com respectivos escores de um a quatro. O escore total pode variar de 16 
(ausência de preocupação), a 64

Os instrumentos utilizados na mensuração de ambos os testes foram: um cronômetro (Cásio, Malaysia), uma cadeira sem apoio de braços e uma trena (Sanny, Brasil).

\section{Intervenção}

A amostra foi submetida a uma intervenção com o protocolo Fisiomotricidade criado por Castro ${ }^{6}$ sendo realizada em grupo e composto de níveis progressivos de intensidade e complexidade distribuídos por três meses ininterruptos, numa frequência de duas vezes por semana, durante 50 minutos por sessão.

O protocolo de exercícios teve início com movimentos de pequena amplitude articular e leve contração isométrica local evoluindo para global, sempre associado a um padrão expiratório, sendo distribuído em quatro níveis, a saber:

Nível I (1a semana) - exercícios de consciência corporal, associados à compreensão dos padrões respiratórios;

Nível II ( $2^{\underline{a}}$ a $4^{\underline{a}}$ semana) exercícios leves, com movimentos de mínima amplitude com sutil contração isométrica local;

Nível III (5 $5^{\underline{a}}$ a $10^{\underline{a}}$ semana) exercícios leves, envolvendo movimentos de pequena amplitude, contração isométrica global associada à contração isotônica livre (sem carga);

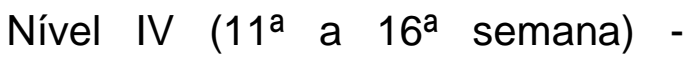
movimentos de grande amplitude (preocupação extrema) ${ }^{11}$.

com contração isométrica global máxima e isotônica com carga, com evolução no nível de complexidade dos movimentos.

\section{Análise estatística}

A análise estatística foi realizada utilizando o programa SPSS 18.0. Na estatística descritiva, as variáveis quantitativas foram apresentadas em mediana, mínimo e máximo. Na análise de correlação entre o medo de cair e o escore do risco de cair utilizou-se a correlação de Spearmann. Para a análise da distribuição normal foi utilizado o teste Shapiro-Wilk e como os dados não apresentaram normalidade, foi utilizado o teste de Wilcoxon na análise intergrupos, sendo considerado significativo $p<0,05$.

\section{Resultados}

Participaram do estudo 30 idosos, todos (100\%) do gênero feminino com mediana de 72 anos, mínimo de 70 e máximo de 75 anos.

Como descrito na tabela 1, a mediana do Tinetti total antes da aplicação do protocolo Fisiomotricidade foi $21(17 ; 25)$ e após três meses de intervenção, a mediana foi de $25(22 ; 28)$. O teste de Wilcoxon foi convertido em um escore $z$ de - 4,80 com uma probabilidade associada de 0,000, demonstrando que a fisiomotricidade acarretou melhoras significativas em relação ao risco de cair nas idosas estudadas. 
Tabela 1. Efeito da Fisiomotricidade sobre o risco de cair. São Luís, 2014.

\begin{tabular}{cccc}
\hline Risco de cair & Mediana & Min-máx & $p$ \\
\hline $\begin{array}{c}\text { Antes da } \\
\text { intervenção }\end{array}$ & 21 & $17-25$ & \\
$\begin{array}{c}\text { Depois da } \\
\text { intervenção }\end{array}$ & 25 & $22-28$ & 0,000 \\
\hline
\end{tabular}

Com relação ao medo de cair, a mediana dos escores foram de 30, antes da intervenção (Gráfico 1) e 21, após a aolicação do protocolo (Gráfico 2), com z de -
4,57 e $p=0,000$, indicando que também houve melhoras significativas após o protocolo de fisiomotricidade

Gráfico 1 - Efeito da Fisiomotricidade quanto ao medo de cair de idosas antes do protocolo de fisiomotricidade. São Luís, 2014.

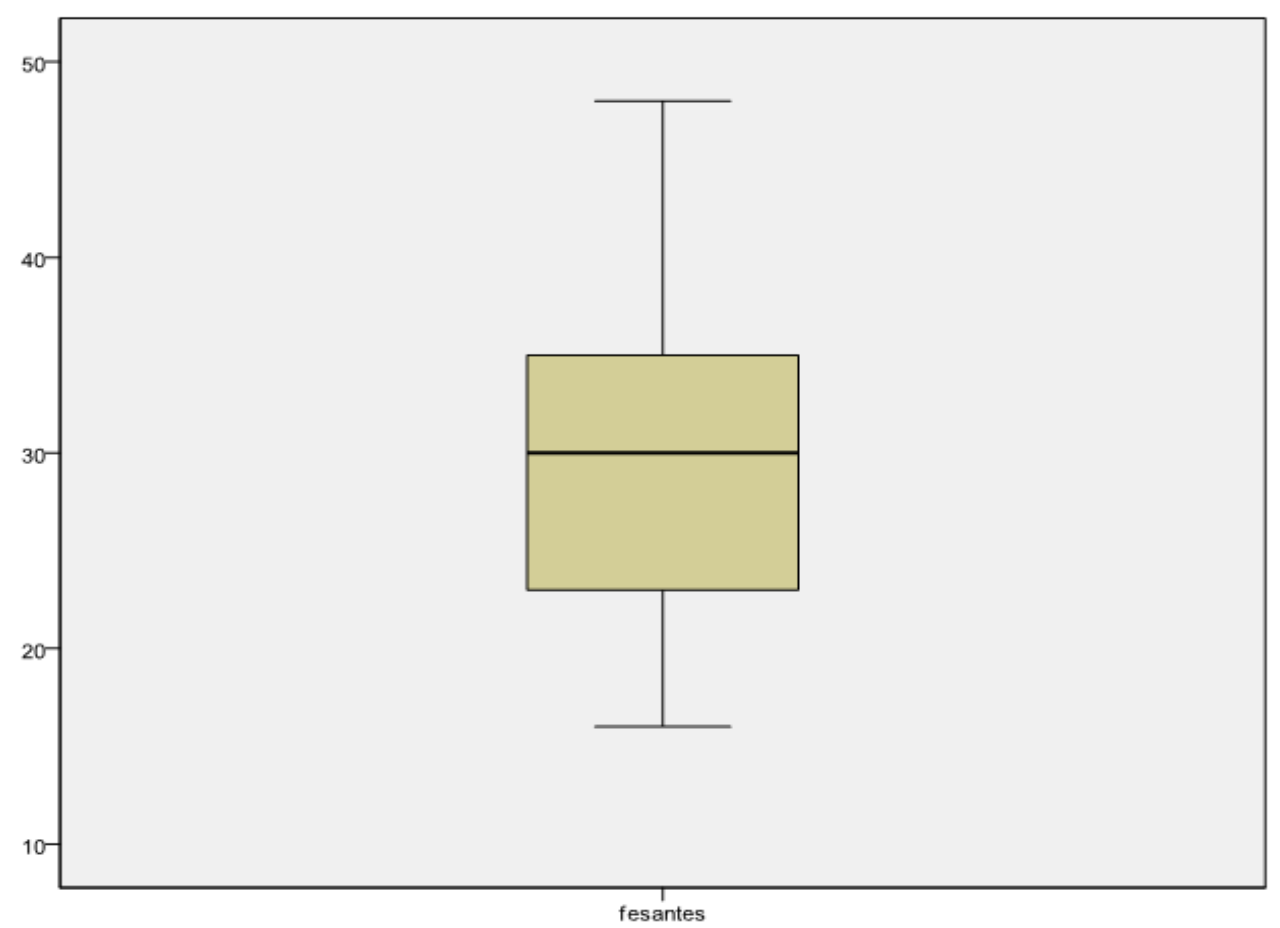


Gráfico 2 - Efeito da Fisiomotricidade quanto ao medo de cair de idosas após o protocolo de fisiomotricidade. São Luís, 2014.

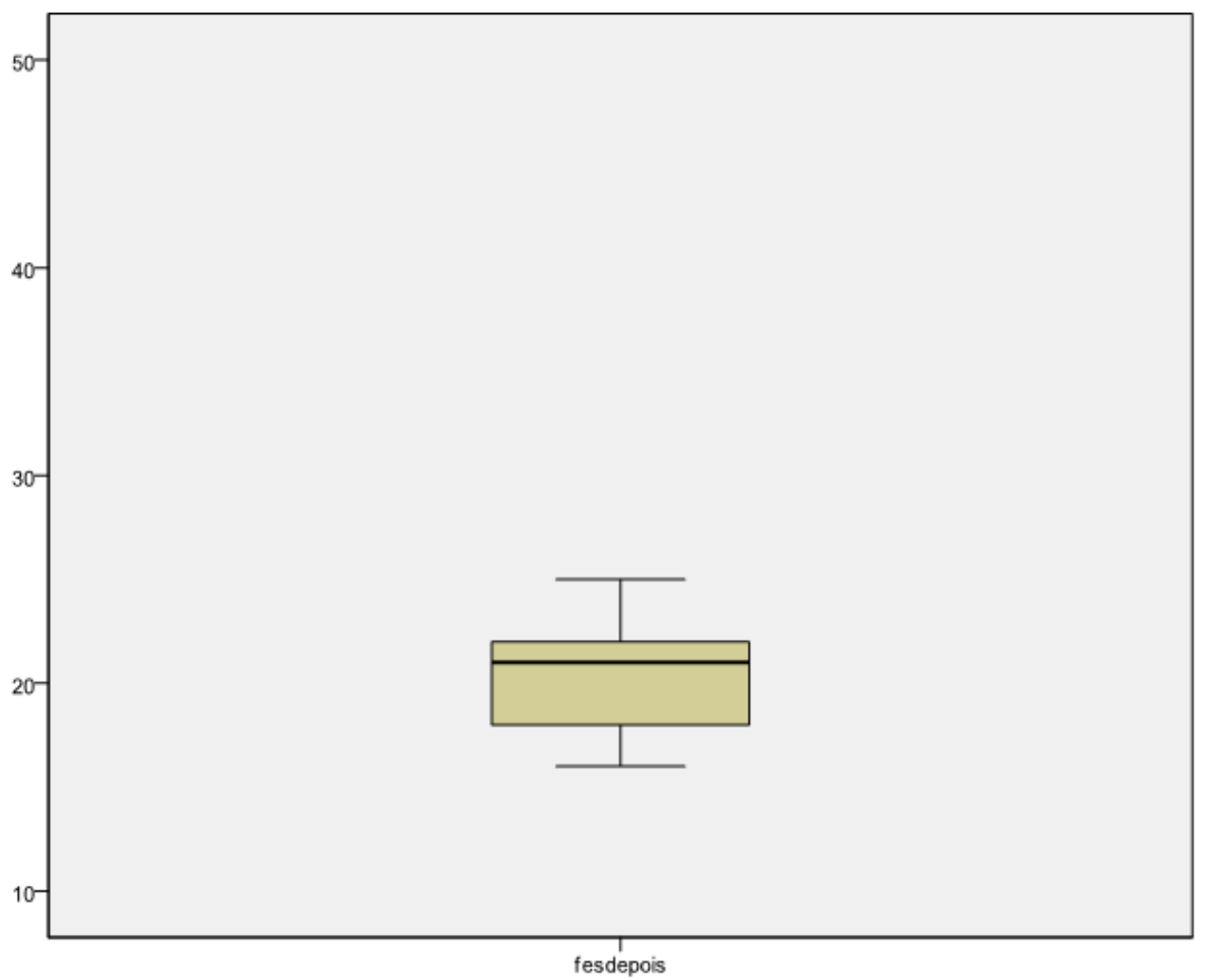

\section{Discussão}

A presença de idosas do sexo feminino no grupo estudado pode ser conxtextualizada pelo processo de feminização na velhice, demonstrando que a população feminina cresce em uma velocidade maior que a masculina, provavelmente por um maior índice de mortalidade no gênero masculino e maior expectativa de vida na população feminina ${ }^{12}$.

A escala de Tinetti vem sendo amplamente utilizada por sua facilidade de aplicação em triagens em idosos na tentativa de identificar possíveis caidores, já que a mobilidade e a marcha, quando alteradas, constituem um dos mais significativos fatores de risco para quedas.Os autores acrescentam que alguns aspectos da locomoção deficiente (iniciação da caminhada, virar, parar) são altamente relacionados com quase todo tipo de queda ${ }^{13}$.

Após serem submetidas a um protocolo de exercícios durante três meses, observou-se um ganho nas valências de equilíbrio e marcha, o que segundo a escala de Tinetti, representa uma diminuição no risco de cair.

Mesmo sabendo que a queda possui etiologia multifatorial, e que sua causa não deve ser associada a apenas um fator isoladamente, estudos apontam que idosos com maior risco de quedas são aqueles com menores índices de agilidade e equilíbrio dinâmico.

Estudo demonstrou que a participação regular do idoso em programas de exercícios físicos 
moderados pode diminuir o risco de quedas, bem como de lesões associadas, enquanto alertam que atividades vigorosas podem aumentar tal risco ${ }^{14}$. Considera-se que existe uma relação em forma de "U" entre a ocorrência de quedas e a prática de exercícios físicos, e alertam que praticar pouco exercício físico ou demasiadamente também podem ser considerados fatores de risco para quedas ${ }^{15,16}$.

Em concordância com os autores supracitados no presente estudo, a modalidade realizada pelos idosos foi a Fisiomotricidade, protocolo cinesioterapêutico de baixo e médio impacto que demonstrou sua eficácia quanto à melhora do equilíbrio e provável redução na ocorrência de quedas.

Marques $^{17}$ realizou um estudo descritivo e prospetivo com a duração de três anos sobre a influência do exercício físico na aptidão física e no equilíbrio de idosos. Os resultados obtidos durante este tempo demonstraram que houve melhoria na aptidão física funcional e no equilíbrio dos idosos.

Ao comparar os fatores de risco para quedas em idosas com diferentes níveis de atividade física, indicou-se que o risco de quedas é maior em idosas com menor nível de atividade e que isto acontece mesmo em relação a variáveis que não são afetadas diretamente pelo exercício, como a auto-percepção de saúde e medo de cair ${ }^{12}$.

Os estudos apontam que os idosos sedentários possuem menor mobilidade e maior propensão a quedas quando comparados a idosos que praticam atividade física regularmente, o que torna possível inferir que uma melhor capacidade funcional relaciona-se com uma menor ocorrência de quedas, sendo essa relação mais significativa no grupo de idosos sedentários ${ }^{18}$.

A melhora no equilíbrio e marcha alcançados após a intervenção com o protocolo de fisiomotricidade pode indicar que a prática de uma atividade física regular para 0 idoso, preferencialmente de médio impacto, pode colaborar na melhora do padrão de controle postural, minimizando acidentes como a queda $^{19}$.

Também foi analisado o efeito da fisiomotricidade na diminuição do medo de cair das idosas. A preocupação em cair identificada no pré-teste, em todas as idosas que participaram do estudo (pontuação acima de 16 pontos), pode ser entendida pelo impacto causado pelo envelhecimento, seja ele físico ou psicológico. Sugerem-se que a diminuição do equilíbrio, da força, da mobilidade e da autoconfiança associadas ao envelhecimento diminuem a auto-confiança fazendo com que o medo de cair esteja presente mesmo naqueles indivíduos que não têm histórico de quedas $^{12}$.

Após a intervenção observou-se uma melhora significativa no índice de equilíbrio e marcha no grupo analisado, como também no medo de cair. Este resultado pode sugerir que 0 protocolo utilizado pode ser uma ferramenta para a melhora também da socialização dos idosos, já que o medo de cair pode estar associado à limitação de atividades funcionais e de vida diária.

Sobre este aspecto, foi investigada a restrição de atividades 
por medo de cair em idosos comunitários e concluiu que esta restrição de fato existe e pode acarretar efeitos negativos na capacidade funcional e nos aspectos psicológicos de idosos. Além disso, observou-se que os idosos que relataram restrição de atividades por medo de cair são mais lentos na marcha, apresentam pior desempenho na realização das Atividades Instrumentais de Vida Diária (AIVD), maior número de doenças e autorrelato de depressão ${ }^{20 .}$

Desta forma, o medo de cair não pode ser negligenciado em pesquisas com idosos, visto que pode trazer consequências tão nefastas quanto a própria queda, em função das limitaçãoes funcionais por ele impostas. Sobre este aspecto, um estudo com 28 idosos inseridos no Programa de Atividades Físicas para Terceira Idade do Centro de Desportos da Universidade Federal de Santa Catarina indicou que, entre os idosos que caíram, $78,57 \%$ relataram medo de cair novamente e $45,85 \%$, assumiram que modificaram seus hábitos relacionados a Atividades de Vida Diária (AVD) em virtude da queda ${ }^{21}$. De forma similar, uma pesquisa realizada com 72 idosos, constatou que $88,5 \%$ tem medo de cair novamente, $26,9 \%$ relataram abandono de atividades e 23,1\% alteraram seus hábitos devido a quedas anteriores ${ }^{22}$.

Aponta-se ainda que não está claro se idosos com medo de cair reduzem a velocidade da marcha para evitar a queda ou se reduzem as atividades, como adaptação ao medo de cair, resultando em alteração na marcha.
O que se sabe é que as medidas dos parâmetros da marcha, como a velocidade da marcha, são indicadores fiéis da mobilidade no idoso e têm capacidade preditiva em relação ao medo de cair, além de serem fortes preditores de eventos adversos, como futuras quedas, declínio funcional e até mesmo a mortalidade ${ }^{23}$.

\section{Conclusão}

Com base nos resultados deste estudo pode-se sugerir que a intervenção do protocolo fisiomotricidade durante três meses provocou melhoras significativas nas variáveis medo e risco de cair das idosas analisadas, apesar de não ter sido apontada correlação entre as duas variáveis.

Conclui-se assim, que a fisiomotricidade pode ser um instrumento adequado para melhorar valências físicas a exemplo de equilíbrio, mobilidade e marcha em idosos. No entanto, sugere-se que sejam feitos novos estudos clínicos com presença de grupo controle e maior número de participantes.

\section{Referências}

1. Alves NB, Scheicher ME. Equilíbrio postural e risco para queda em idosos da cidade de Garça, SP. Rev. bras. geriatr. gerontol. 2011; 14( 4 ): 763768.

2. Santos GM, Souza ACS, Virtuoso JF, Tavares GMS, Mazo GZ. Valores preditivos para o risco de queda em idosos praticantes e não praticantes de atividade física por meio do uso 
da Escala de Equilíbrio de Berg. Rev. bras. Fisioter.2011; 15 ( 2 ): 95-101.

3. Juliana GAI et al, Ptofobia - o medo de cair em pessoas idosas, Acta Med Port. 2009; 22(1):83-88

4 Almeida P; Neves R. As quedas e o medo de cair em pessoas idosas institucionalizadas, Revista Kairós Gerontologia,12012; 5(5), 27-43.

5 Matsudo SM, Matsudo VKR, Barros TL Neto. Efeitos benéficos da atividade física na aptidão física e saúde mental durante o processo de envelhecimento. Rev Bras Ativ Fis Saúde 2000; 5(2):60-76.

6. Castro KVB, Silva ALS, Lima JMMP, Nunes WJ, Calomeni MR, Silva VF. Fisiomotricidade e limiares de dor: efeitos de um programa de exercícios na autonomia funcional de idosas osteoporóticas. Fisioter. mov. (Impr.) 23( 1 ): 161-172.

7. Mata FAF, Barros ALS, Lima CF. Avaliação do risco de queda em pacientes com Doença de Parkinson. Rev Neurocienc.2008;16/1: 20-24.

8. Karuka Ah, Silva Jam, Navega Mt. Análise da concordância entre instrumentos de avaliação do equilíbrio corporal em idosos. Rev Bras Fisioter. 2011;15(6):460-6

9. Tinetti, M.E.Performance-oriented assessment of mobility problems in elderly patients. J Am Geriatr Soc. 1986;34:119-26

10. Gomes GC. Tradução, adaptação cultural e exame das propriedades de medida da escala "Performance Oriented Mobility Assessment" POMA para uma amostragem de idosos brasileiros institucionalizados [dissertação]. Campinas: Universidade Estadual de Campinas; 2003
11. Camargos FFO, Dias RC, Dias JMD, Freire MTF. Adaptação transcultural e avaliação das propriedades psicométricas da Falls Efficacy Scale - International em idosos brasileiros (FES-I-BRASIL). Rev Bras Fisioter. 2010;14:237-43.

12. Silva LRV, Rodacki ALF, Rebutini VZ, Góes SM, Coelho RW, Stefanello JMF. Efeitos de curto prazo de um programa de imaginação sobre o medo de queda de indivíduos pós acidente vascular encefálico.Motriz: rev. educ. fis. 2013; 19(1):46-54..

13. Silva VF, Matsuura C. Efeitos da prática regular de atividade física sobre o estado cognitivo e a prevenção de quedas em idosos. Fit Perf J. 2002;1(3):39-45.

14. Streit IA, Mazo GZ, Virtuoso JF, Menezes EC, Gonçalves E. Aptidão física e ocorrência de quedas em idosos praticantes de exercícios físicos. Rev Bras Ativ Fis Saúde. 2011;16(4):346-52.

15. Vogel T, Brechat PH, Lepretre PM, Kaltenbach G, Berthel M, Lonsdorfer J. Health benefits of physical activity in older patients: a review. Int J Clin Pract. 2009; 63:303-20.

16. Wright, RL.Implications for Falls Prevention of Lifetime Physical Activity and Control of Gait, Posture and Balance in Older Adults. [Tese]. University of Coventry in collaboration with the University of Worceste, 2009.

17. Marques C. Avaliação da aptidão física e do equilíbrio em idosos. In: XVI Jornadas Internacionais do Instituto Português de Reumatologia. Centro de Congressos de Lisboa, 2008; Lisboa; 2008. 
18. Brito J, Bicho I, Ramos L, Ricardo N, Fernandes R. Aptidão funcional, equilíbrio e ocorrência de quedas em idosos. Revista da Unidade de Investigação do Instituto Politécnico de Santarém, 2013;1(1),247-259..

19. Piccoli JCJ, Quevedo DM, Santos GA, Ferrareze ME, Gluher A. Coordenação global, equilíbrio, índice de massa corporal e nível de atividade física: um estudo correlacional em idosos de Ivoti, RS, Brasil. Rev. bras. geriatr. gerontol. 2012; 15 ( 2 ): 209-222.

20. Dias RC, Freire MTF, Santos EGS, Vieira RA, Dias JMD, Perracini MR. Características associadas à restrição de atividades por medo de cair em idosos comunitários. Rev. bras. fisioter. 2011; $15(5$ ): 406-413.

21. Beck AP, Antes DL, Meurer ST, Benedetti TRB, Lopes MA. Fatores associados às quedas entre idosos praticantes de atividades físicas. Texto contexto - enferm. 20( 2 ): 280-286.

22. Ribeiro A, Souza E, Atie S, Souza A, Schilithz A. A influênciadasquedasnaqualidadedevi dadeidosos. Ciên Saúde Coletiva. 2008 Jul; 13(4):1265-73.

23. Kirkwood Rn, Moreira Bs, Vallone Mldc, Mingoti As, Dias Rc, Sampaio Rf. Step length appears to be astrong discriminant gait parameter for elderly females highly concerned about falls: a cross-sectional observational study. Physiotherapy 2011;97(2):126-31. 